\title{
Discrimination between Mature Vinegars of Different Geographical Origins by NIRS*
}

\author{
Huishan $\mathrm{Lu}^{1, * *}$, Zhengguang $\mathrm{An}^{1}$, Huanyu Jiang ${ }^{2}$, and Yibin Ying ${ }^{2}$ \\ ${ }^{1}$ College of Mechanical Engineering \& Automatization, North University of China, \\ 3 Xueyuan St., Taiyuan 030051, P.R. China \\ ${ }^{2}$ College of Biosystems Engineering and Food Science, Zhejiang University, \\ 268 Kaixuan St., Hangzhou 310029, P.R. China \\ 13934597379@139.com, hyjiang@zju.edu.cn, ybying@zju.edu.cn
}

\begin{abstract}
The feasibility of near infrared spectroscopy (NIRS) for discrimination between mature vinegar of different geographical origins (Taiyuan and Qingxu, China) is presented in this research. NIR spectra were collected in transmission mode in the wavelength range of 800-2500 $\mathrm{nm}$. Qualitative analysis models were developed based on Principal Component Analysis (PCA) and Discriminant Analysis (DA). The prediction performance of calibration models in different wavelength range was also investigated. The best models gave a 96.3\% classification of mature vinegars of the two geographical origins in the range of $800-2500 \mathrm{~nm}$. The results demonstrated that NIRS technique could be used as a rapid method for classification of geographical origin of mature vinegars.
\end{abstract}

\section{Introduction}

With increasing concerns about food products adulteration, falsification of added-value claims and traditional foods protection, food authentication is an essential challenge that must be faced in many different quality control tasks, such as guaranteeing the actual origin of a product and detecting deliberate or accidental adulteration of valuable food components by inferior ingredients. The food industry, regulatory authorities and consumers are all interested in authenticating raw materials and verifying the origin or the specific character of a product in order to promote quality and to assure food safety.

In particular, assurance of the quality and origin of vinegars has become an issue of supreme commercial importance as a means for searching for safety in the vinegar industry and for fighting to curb unfair competition, also taking into account the huge diversity of vinegars available nowadays on the market and the differences in the final sale price depending on a wide range of quality factors. Vinegar is a product resulting from the alcoholic and subsequent acetic fermentation of any fermentable starting material rich in carbohydrates. The final quality and organoleptic properties of vinegar

\footnotetext{
* The paper supported by by Shanxi Youth Science and Technology Research Fund (No. 2009021019-3).

** Corresponding author.
}

D. Li, Y. Liu, and Y. Chen (Eds.): CCTA 2010, Part I, IFIP AICT 344, pp. 72977362011.

(C) IFIP International Federation for Information Processing 2011 
are determined by a profound chemical complexity conditioned by both the raw material employed and the particular elaboration process used. Traditional and selected vinegars are produced by slow acetification methods, which usually require long periods of maturation in wood to reach the desired acetic degree. These traditional elaboration processes, involving simultaneous acetification and ageing, produce excellent quality vinegar, but at the expense of increasing production time and cost, which is used to justify their premium price. Therefore, reliable methods are required for quality and economical reasons in order to objectively differentiate vinegars according to their origin and commercial type, and thus provide real protection to superior quality products and to ensure vinegar authenticity. Flavour and aroma are some of the most important factors in the determination of vinegar character and quality. A large variety of compounds covering a wide range of volatilities and concentrations are responsible for the sensorial complexity of vinegars. The volatile fraction of any specific vinegar, and thus its aromatic composition, is significantly influenced by many factors, the main sources of variation being the particular raw material used, the acetification system employed for its production, and eventually wood ageing. Therefore, since the volatile profile of vinegar represents a fingerprint of the sample, it is reasonable to assume that a classification approach based on the analysis of volatile components would be an efficient tool for evaluating vinegar authenticity. In fact, a number of previous studies have described the effective application of the analysis of volatile compounds for the characterization and differentiation of different vinegars. Although it should be noted that this is only a feasibility study, the promising results obtained justify a similar approach to be considered in future in order to better evaluate its actual performance and to broaden the field of application to a wider range of vinegar types.

\section{Materials and Method}

\subsection{Samples}

In this research, a total of two geographical origins of mature vinegars were obtained in different region. Thirty six bottles of mature vinegar samples of "Donghu" brand were from Qingxu, and thirty six bottles of "Ninghuafu" brand from Taiyuan. In all, 12 samples were of 1 year age, 12 samples were of 3 year age, 12 samples were of 5 year age in "Donghu" Group and "Ninghuafu" Group, respectively. Before the experiment, the mature vinegar samples were stored in the laboratory at a constant temperature of $25 \pm 1{ }^{\circ} \mathrm{C}$ for more than $48 \mathrm{~h}$ to have an equalization room temperature. The samples were all original vinegar liquid without dilution. Sixty mature vinegar samples were used in the calibration set, whereas, 12 samples ( 2 for each age of each group) were selected as the validation set.

\subsection{Spectral Measurements}

Samples taken from freshly opened bottles of mature vinegar were scanned in transmission mode using a commercial spectrometer Nexus FT-NIR (Thermo Nicolet Corporation, Madison, WI,USA) which was equipped with an interferometer, an InGaAs detector, and a broad band light source (Quartz Tungsten Halogen, 50 W). 
Samples were scanned in a $1 \mathrm{~mm}$ optical path-length rectangular quartz cuvette with air as reference at room temperature. NIR spectra were collected using OMNIC software (Thermo Nicolet Corporation, Madison, WI, USA) and stored in absorbance format. The spectral range was from 800 to $2500 \mathrm{~nm}$, the mirror velocity was $0.9494 \mathrm{~cm} \mathrm{~s}-1$, and the resolution was $16 \mathrm{~cm}-1$ in this work. The spectrum of each sample was the average of 32 successive scans.

\subsection{Chemometrics and Data Analysis}

Chemometrics analyses including PCA and DA were performed using a commercial software package (TQ Analyst software, Thermo Electron Corp., Madison, Wisc.). Spectra were exported from OMNIC software in absorbance format to TQ Analyst software before being analyzed.

\section{Principal Component Analysis (PCA)}

In this work, PCA was used to reduce the dimensionality of the NIR transmission spectra recorded on mature vinegar samples and extract the most useful and relevant information. PCA transforms the original independent variables (wavelengths) into new axes and calculates the principal components (known as PCs) as new variables by relevant algorithm to replace the original data (Mouazen et al., 2006). The PCs are orthogonal, so the data presented on the new axes are uncorrelated with each other $(\mathrm{Xu}$ and Shao, 2004). The PCs account as much as possible for the variability in the original variables (Cozzolino et al., 2003). Each spectrum will have its own unique set of scores; therefore, a spectrum can be represented by its PCA scores in the factor space instead of by intensity in the wavelength space (Park et al., 2003). The scores of such PCs replace the original spectra data to be used for further modeling.

\section{Discriminant Analysis (DA)}

DA was used in this work to build a classification model to discriminate "Ninghuafu", and "Donghu" mature vinegar samples based on predefined classes and determine the percentage of correct classifications. Because the first ten PCs cover most of the variation ( $>99.9 \%$ of the total variance) of the raw spectral data, the first ten PCs attained through PCA calculation of the spectral data were employed for DA. In TQ Analyst, discrimination of the groups consists of calculating the Mahalanobis distance of a sample from the centers of gravity of the considered groups; one can then clarify the properties that distinguish the different groups. A sample is assigned to the group that has the shortest Mahalanobis distance to it, with which it is considered to be the most similar.

\section{Results and Discussion}

\subsection{Spectral Analysis}

Figure 1 shows the average NIR absorbance spectra of "Ninghuafu" and "Donghu" samples without any preprocessing. No obvious spectral differences could be observed 
H. Lu et al.

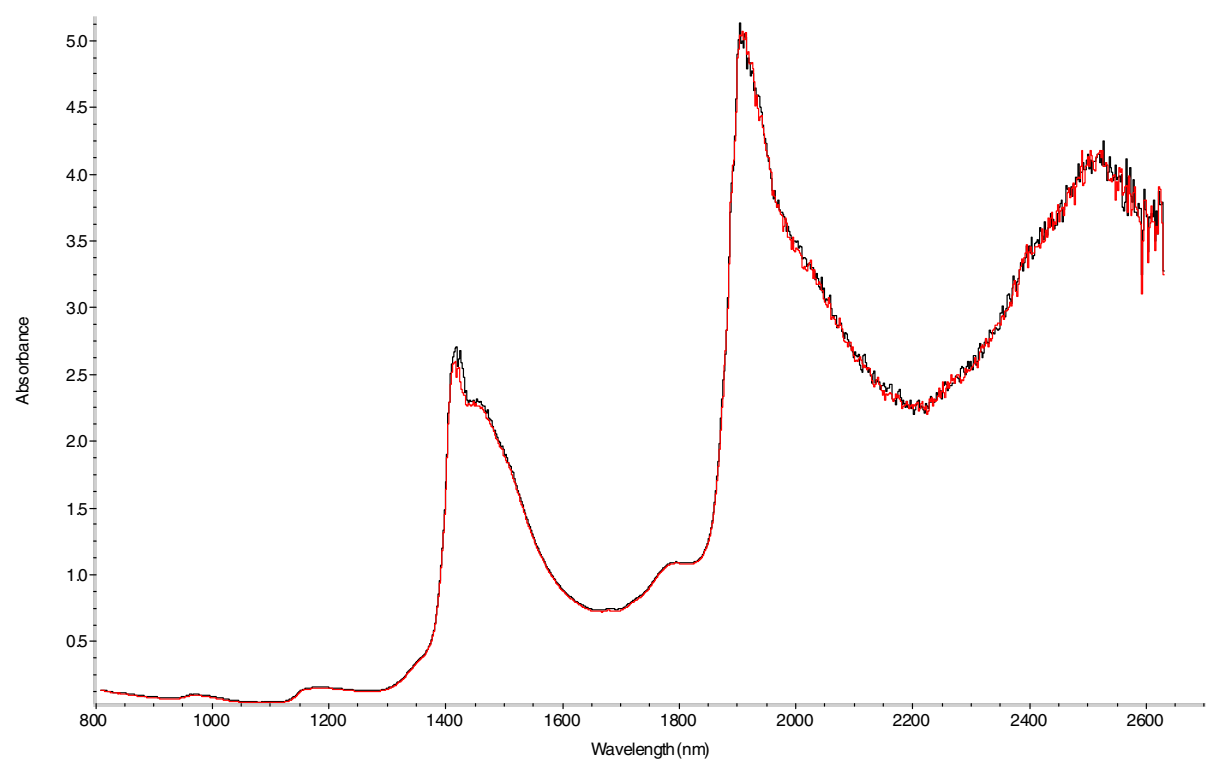

Fig. 1. Average NIR spectra of 36 "Ninghuafu" and 36 "Donghu" samples without any preprocessing

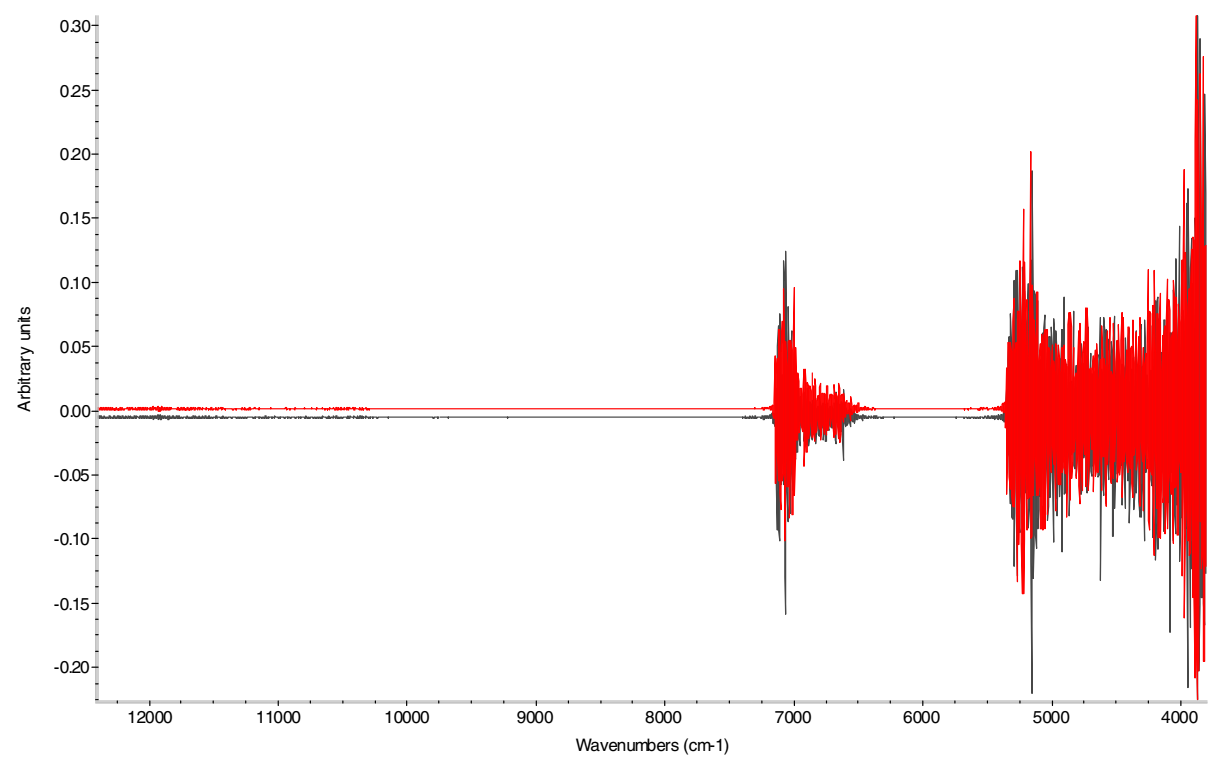

Fig. 2. Average second-derivative spectra of 36 "Ninghuafu" and 36 "Donghu" samples 
between the two spectra, and the spectra are highly overlapped except in the regions 940-1025 nm, $1125-1360 \mathrm{~nm}, 1670-1840 \mathrm{~nm}$, and 2000-2100 nm, where "Ninghuafu" is a little higher than "Donghu", while at $1390-1435 \mathrm{~nm}$ as well as $2380-2480 \mathrm{~nm}$, "Donghu" is a little higher than "Ninghuafu". All spectra have intense absorption bands at $1450 \mathrm{~nm}$, related to the first $\mathrm{O}-\mathrm{H}$ overtone, and at $1900-1950 \mathrm{~nm}$, related to the combination of stretch and deformation of the $\mathrm{O}-\mathrm{H}$ group in water. The small absorption band at $1690 \mathrm{~nm}$ might be related to the - $\mathrm{CH} 3$ stretch first overtone or $\mathrm{C}-\mathrm{H}$ groups in aromatic compounds, at $1782 \mathrm{~nm}$ related to the $\mathrm{C}-\mathrm{H}$ stretch first overtone, at 2266 $\mathrm{nm}$ likely with C-H combination bands of methanol, and at $2302 \mathrm{~nm}$ with combination band of $\mathrm{C}-\mathrm{H}$ stretch and deformation of $\mathrm{C}-\mathrm{H}$ from the - $\mathrm{CH} 2$ group (Yu et al., 2007; Cozzolino et al., 2003; Yan et al., 2005; Lu, 2007; Dambergs et al., 2002). Figure 2 shows the averaged second-derivative spectra of mature vinegar from the two geographical origins, from which we can observe that the averaged second-derivative spectrum of "Ninghuafu" is higher than that of "Donghu" except for an overlap at 1900-1950 nm due to intense absorbance of water. There are obvious differences between the three spectra that are highly overlapped in figure 1, which demonstrate that features of the two spectra are enhanced after preprocessing with second derivatives.

\subsection{Principal Component}

All the spectra of 36 "Ninghuafu" samples, 36 "Donghu" samples were preprocessed with multiplicative signal correction (MSC) and second derivatives to reduce spectral variations and enhance features of the spectra before PCA. The first ten PCs account for $99.475 \%$ of the variation in the spectra, and are used to make differentiation clearer.

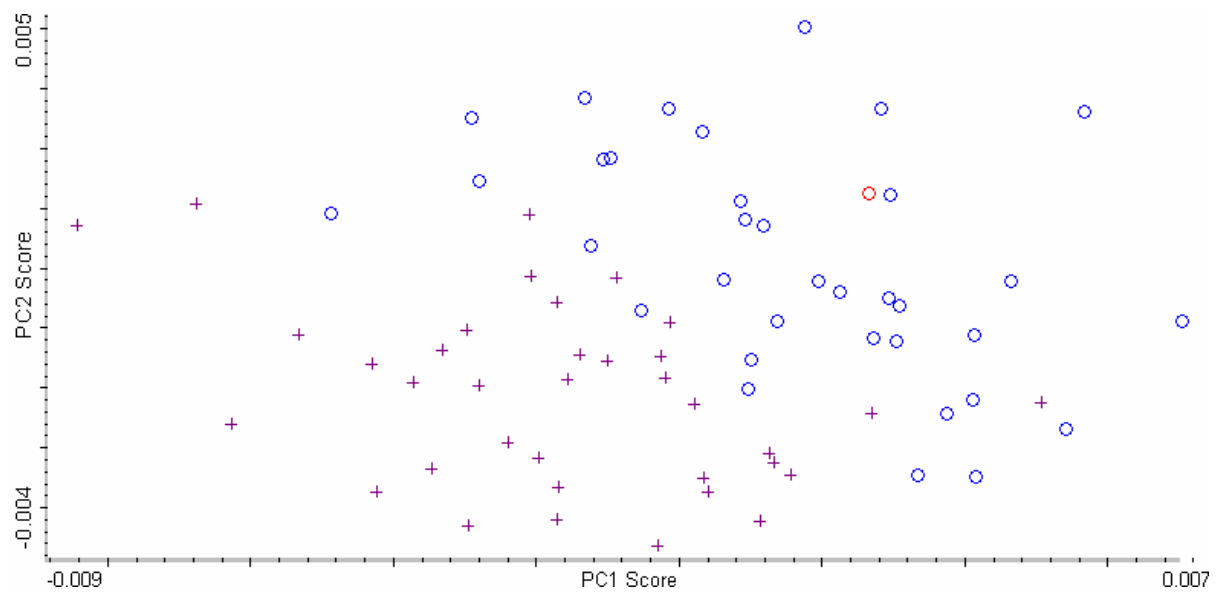

Fig. 3. Two-dimensional score plot for "Ninghuafu" samples and "Donghu" sample

From figure 3, we can see that the samples were divided into two groups. The separation of "Ninghuafu" from the other was clear, but with some overlapped samples. However, the classification trends are obvious in this figure, and samples of the three 
brands can be separated. Figure 3 shows classification of "Ninghuafu" and "Donghu" using discriminant analysis method with obvious boundaries that include an overlapping of the two brands. It can be observed in this figure that the "Ninghuafu" and "Donghu" samples had both positive and negative scores.

From the plot, we can see that "Ninghuafu" and "Donghu" were separated perfectly with 5 overlapped samples. This result suggested that discrimination among the two brands was possible, but as the result was not ideal and PCA only represented a potential ability to discriminate the samples, discriminant analysis was employed in order to obtain a better classification.

\subsection{Discriminant Analysis}

As a rule, the DA procedure supplies two kinds of functions: discriminant functions, which provide the separation among groups, typically in one- or two- dimensional space, and classification functions, which are used to assign a new case into one of the known groups (Nicolas et al., 2000; Yu and Ren, 1999). In this work, the former function was used to discriminate the two brands. Sixty samples (30 "Ninhuafu" and 30

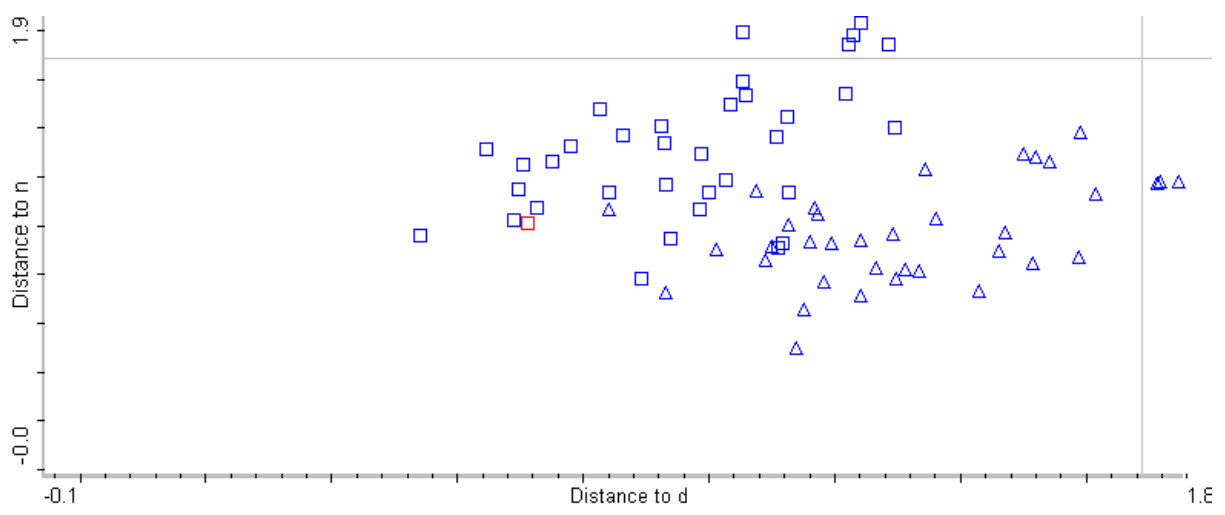

Fig. 4. Classification of "Ninghuafu" and "Donghu" using discriminant analysis method

Table 1. The result of discriminant analysis method

\begin{tabular}{ccccc}
\hline Group & $\begin{array}{c}\text { Age (Number of } \\
\text { samples) }\end{array}$ & $\begin{array}{c}\text { Number of samples } \\
\text { Calibration } \\
\text { set }\end{array}$ & $\begin{array}{c}\text { Validation } \\
\text { set }\end{array}$ & $\begin{array}{c}\text { Correctly } \\
\text { Classified }\end{array}$ \\
\hline \multirow{2}{*}{ Donghu } & $\begin{array}{l}\text { 1-year (10) } \\
\text { 3-year (10) }\end{array}$ & 30 & 6 & $96.3 \%$ \\
& $\begin{array}{l}\text { 5-year (10) } \\
\text { 1-year (10) }\end{array}$ & & & \\
3-year (10) & 30 & 6 & $95.6 \%$ \\
\hline
\end{tabular}


"Donghu") were selected as the calibration set, and the 12 samples (6 "Ninhuafu" and 6 "Donghu") were used as the validation set. And the details were listed in Table 1. Plots of the Mahalanobis distances of all samples to "Ninhuafu" and to "Donghu" are shown in figure 4. It can be seen in these plots that the two brands were divided into two clusters. On the whole, a good result was obtained, with a classification accuracy of 95.9\%. The result indicates that DA was effective for the discrimination of the two brands and produced a good classification model.

\section{Conclusions}

The result obtained in this work suggests that NIR spectroscopy, together with chemometrics methods such as DA classification models based on PCA, is a powerful technique to classify objects, and it was effective for discriminating mature vinegar samples from different different geographical origins ("Ninghuafu" from Taiyuan, and "Donghu" from Qingxu). In this case, the best classification, with accuracy up to $96.3 \%$, was attained by DA based on sample spectra pretreated with second derivatives. This technique can acquire enough information to offer an ideal result, avoiding time-consuming, costly, and laborious chemical and sensory analysis. This result demonstrates that NIR spectroscopy has a good potential for use as an alternative technique for developing an accurate, rapid detector to discriminate mature vinegar from geographical origins. It is unknown which components account for the variation and act in the discrimination, so further study of the chemical constituents in mature vinegar should follow. At the same time, the work reported here is a feasibility study and requires further development with considerably more samples of other brands and varieties.

\section{Acknowledgement}

The authors gratefully acknowledge the financial support provided by Shanxi Youth Science and Technology Research Fund (No. 2009021019-3).

\section{References}

1. Mouazen, A.M., Karoui, R., De Baerdemaeker, J., Ramon, H.: Classification of soils into different moisture content levels based on NIS-NIR spectra. ASABE Paper No. 061067 (2006)

2. Xu, L., Shao, X.G.: Methods of Chemometrics. Science Press, Beijing (2004)

3. Cozzolino, D., Smyth, H.E., Gishen, M.: Feasibility study on the use of visible and near-infrared spectroscopy together with chemometrics to discriminate between commercial white wines of different varietal origins. J. Agric. Food Chem. 51(26), 7703-7708 (2003)

4. Park, B., Abbott, J.A., Lee, K.J., Choi, C.H., Choi, K.H.: Near-infrared diffuse reflectance for quantitative and qualitative measurement of soluble solids and firmness of delicious and gala apples. Trans. ASAE 46(6), 1721-1731 (2003) 
5. Yu, H.Y., Zhou, Y., Fu, X.P., Xie, L.J., Ying, Y.B.: Discrimination between Chinese rice wines of different geographical origins by NIRS and AAS. European Food Res. Tech. 225(3-4), 313-320 (2007)

6. Yan, Y.L., Zhao, L.L., Han, D.H., Yan, S.M.: Basic and Application of Near-Infrared Spectral Analysis. China Light Industry Press, Beijing (2005)

7. Lu, W.Z.: Modern Near Infrared Spectroscopy Analytical Technology, 2nd edn. Petrochemical Press, Beijing (2007)

8. Dambergs, R.G., Kambouris, A., Francis, I.L., Gishen, M.: Rapid analysis of methanol in grape-derived distillation products using near-infrared transmission spectroscopy. J. Agric. Food Chem. 50(11), 3079-3084 (2002)

9. Niu, X.Y., Yu, H.Y., Ying, Y.B.: The Application Of Near-Infrared Spectroscopy And Chemometrics To Classify Shaoxing Wines From Different Breweries. Transactions of the ASABE 51(4), 1371-1376 (2008) 